\title{
Meta
}

Journal des traducteurs

Translators' Journal

\section{DECONINCK, Julie, Humblé, Philippe, SEPP, Arvi et al., dir. (2017) : Towards Transcultural Awareness in Translation Pedagogy. Zurich : LIT Verlag, 271 p.}

\section{Kornebari B. Kumbe}

Volume 64, numéro 1, avril 2019

URI : https://id.erudit.org/iderudit/1065343ar

DOI : https://doi.org/10.7202/1065343ar

Aller au sommaire du numéro

Éditeur(s)

Les Presses de l’Université de Montréal

ISSN

0026-0452 (imprimé)

1492-1421 (numérique)

Découvrir la revue

Citer ce compte rendu

Kumbe, K. B. (2019). Compte rendu de [DEConinCK, Julie, HUMBLÉ, Philippe, SEPP, Arvi et al., dir. (2017) : Towards Transcultural Awareness in Translation Pedagogy. Zurich : LIT Verlag, 271 p.] Meta, 64(1), 298-300.

https://doi.org/10.7202/1065343ar d'utilisation que vous pouvez consulter en ligne. 
difficult to find information on this type of activity. I was hoping that a book of this size would provide a comprehensive look at roles and techniques for the actual work of translation and editing that happens when a team collaborates, but there were only a few small examples. It seems that the goal was to highlight practices and introduce some theory, rather than concentrate on the real processes of collaboration.

However, the book does provide general insights for those seeking to learn about collaborating with authors on a translation, including working with multiple languages. It also provides information about using online tools for collaboration and crowdsourcing. I found it fairly easy to read except for the last chapter, which seemed somewhat disjointed. As with all Bloomsbury publications, the book is beautifully bound, with clear type. The chapters are arranged in logical sections which progress from the history of collaborative translation to actual contemporary practices. I feel that the book would be most valuable to translators and groups of translators intending to work mainly with a single author or type of literature.

LiAnE Johnston Grant Université de Montréal, Montréal, Canada

\section{NOTES}

1. BRUNI, Leonardo (1420-1426/2004): De interpretatione recta [De la traduction parfaite]. In: Paolo Viti, ed. Sulla perfetta traduzzione [Sur la traduction parfaite]. Naples: Liguori Editore.

\section{REFERENCES}

BRUNI, Leonardo (1420-1426/2004): De interpretatione recta. In: Paolo Viti, ed. Sulla perfetta traduzzione. Naples: Liguori Editore.

Cordingley, Anthony, ed. (2013): Self-Translation: Brokering Originality in Hybrid Culture. London/New York: Bloomsbury Academic.

JiméneZ-Crespo, Miguel A. (2017): Crowdsourcing and Online Collaborative Translations. Amsterdam/Philadelphia: John Benjamins.

VITI, Paolo (2004): Sulla perfetta traduzzione [Sur la traduction parfaite]. Naples: Liguori Editore.

Deconinck, Julie, Humblé, Philippe, Sepp, Arvi et al., dir. (2017): Towards Transcultural Awareness in Translation Pedagogy. Zurich: LIT Verlag, $271 \mathrm{p}$.

L'ouvrage Towards Transcultural Awareness in Translation Pedagogy, publié sous la direction de Julie Deconinck, Philippe Humblé, Arvi Sepp et Hélène Stengers, constitue le numéro douze de la collection «Representation - Transformation» dirigée par Michaela Wolf. Le livre regroupe des perspectives théoriques et des idées pratiques sur la dimension culturelle en pédagogie de la traduction. Les auteurs proposent des analyses empiriques et didactiques aussi diverses qu'étendues sur la conception des cours ainsi que sur la docimologie en traduction. Ils partagent le postulat selon lequel les enseignants, dans un cours de traduction, doivent sensibilise $r$ les étudiants à la négociation et la prise en compte des différences culturelles. L'ouvrage renferme une introduction, douze chapitres répartis en trois parties, les notices biographiques des auteurs et deux index.

Rédigée par les quatre directeurs, tous enseignants de traduction à la Vrije Universiteit de Bruxelles en Belgique, l'introduction du livre présente les idées fondamentales qui sous-tendent les perspectives développées par les différents auteurs des douze chapitres. Ces enseignantstraductologues soutiennent le point de vue selon lequel toute traduction, quel que soit le genre ou le contexte, relève essentiellement et fondamentalement de la communication entre des cultures. Par conséquent, ils postulent que dans un cours de traduction, les étudiants doivent être sensibilisés au fait que tout texte à traduire est imprégné d'éléments culturels et que l'analyse de l'écart culturel entre la langue source et la langue cible constitue une condition nécessaire pour la réussite de toute activité de traduction. Ils notent que si une telle affirmation paraît évidente, les différentes approches employées pour enseigner la traduction des éléments culturels ne sont pas mises en évidence ou ces approches sont sousprésentées. C'est dans le but de combler cette lacune que les directeurs ont compilé un certain nombre de perspectives théoriques et d'expériences d'enseignement sur le sujet suivant: «comment aborder la dimension culturelle dans la pédagogie de la traduction» (p. 1; notre traduction). Ainsi, l'ouvrage fait état d'une diversité de perspectives théoriques provenant de différents paradigmes et disciplines et témoignant de plusieurs situations d'enseignement de la traduction et de la culture.

La première partie, intitulée Theoretical Reflections on Translation Pedagogy and Transculturalism, est composée de quatre chapitres. Dans le premier chapitre qui porte le titre The Position of Translation Training: Another Success Story?, José Lambert (Université de Louvain et Université fédérale de Ceará) jette la lumière sur la distinction entre l'institutionnalisation de la traduction et l'institutionnalisation de la traductologie, et estime que cette dernière représente un moment charnière dans l'histoire des universités et de l'univers des connaissances. Lambert soulève la problématique liée au concept de translation studies tel qu'il a été conçu par Holmes (1972). Il attire également 
l'attention sur les limites de l'expression translation $\underline{\text { studies, d'un point de vue terminologique, par }}$

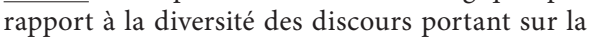
traduction et juge que l'évolution disciplinaire nécessitera une interaction entre les différents modèles de la traductologie.

Dans le chapitre deux, Towards a Culturebased Paradigm for Translator Training, Jan Walravens (Université Libre de Bruxelles) soutient l'idée selon laquelle la traduction est fondamentalement une activité interculturelle. Walravens affirme également que la traduction n'est jamais sans engagement et que la traduction est inévitablement visible puisque le traducteur est toujours engagé dans une sorte de discours politique. L'auteur propose que cette visibilité inévitable de la traduction soit présentée de manière claire dans les programmes de formation. En outre, Walravens examine la multidisciplinarité de la traductologie et des études interculturelles, puis il remarque que l'interdisciplinarité est à l'origine du chevauchement de plus en plus grand entre ces deux disciplines sur les plans théorique et méthodologique.

Dans le chapitre trois intitulé Translation as Bridge-building and Transculturation, Kanavillil Rajagopalan de l'Université d'État de Campinas s'interroge sur la notion de traduction comme transfert de sens d'une culture à une autre. Il réfute l'idée de concevoir une culture donnée comme une entité distincte et argue plutôt que la mondialisation mène aux contacts des cultures et des langues avec pour résultat l'hybridation et la transculturation. En outre, Rajagopalan estime que la discipline anthropologique et la pratique de la traduction ont en commun le but de renforcer la compréhension interculturelle, et juge que la traduction relève tout d'abord de l'anthropologie, puis, en deuxième lieu, elle est une question linguistique.

Elżbieta Tabakowska de l'Université Jagiellonne de Cracovie insiste, dans le chapitre quatre intitulé Grammar, 'Cultural Meaning' and Translation Pedagogy, que la compétence de traduction comprend forcément la grammaire dans le sens de charge culturelle portée par les unités de langue. En s'appuyant sur la théorie cognitiviste, Tabakowska avance l'idée que les structures grammaticales, et non seulement le lexique et les expressions idiomatiques, comportent des sens culturels. Ces aspects grammaticaux relatifs à l'ethnolinguistique doivent être portés à l'attention des traducteurs, insiste Tabakowska. Elle bâtit son argument sur deux études de cas: un extrait d'un roman polonais intitulé Pod Mocnym Aniotem de Jerzy Pilch (2001) traduit sous le titre The Mighty Angel (2010) et une publicité commerciale.

La deuxième partie de l'ouvrage a pour titre Transculturalism in Translation Teaching et contient quatre chapitres portant sur des activités d'apprentissage pratiquées dans des cours de traduction. Natalia Kaloh Vid et Vlasta Kučiš, auteures du premier chapitre intitulé Teaching Translation in the Context of Culture and Ideology, décrivent une activité destinée à sensibiliser les étudiants de traduction à l'Université de Maribor aux fondements idéologiques et aux spécificités culturelles de slogans et de publicités en anglais et en allemand. Ces étudiants étaient capables de porter un regard critique sur les traductions slovènes de ces publicités et de proposer des traductions adéquates le cas échéant, grâce à l'analyse critique du discours (ACD) de van Dijk et à la théorie du skopos de Vermeer, expliquent Vid et Kučiš.

Dans le deuxième chapitre, Teaching Translation in Banking and Finance: Cross-cultural Aspects, Natalia Naydenova (Université russe de l'Amitié des Peuples) argumente que la pédagogie doit mettre l'accent sur la sensibilisation interculturelle dans les cours de traduction spécialisée destinés à former des traducteurs qui travailleront dans un contexte international. L'auteure présente le plan de cours d'un module conçu dans le domaine bancaire et financier dont l'objectif vise à mettre en évidence la différence entre les cultures anglo-saxonne et russe. Naydenova fournit des exercices tirés de la version pilote du module en question et souligne l'importance de ces composantes interculturelles pour ses étudiants.

Contrairement aux auteures des deux chapitres précédents centrés sur des cours de traduction technique, Judith Woodsworth de l'Université Concordia décrit des activités pratiquées lors d'un cours de traduction littéraire du français vers l'anglais dans le chapitre trois intitulé Transposing Voices: Teaching Interculturalism in a Literary Translation Course. D'abord, les étudiants mènent un entretien avec un locuteur d'une troisième culture. Ensuite, en s'appuyant sur l'entretien, les étudiants écrivent une histoire en anglais. Grâce à ces activités, remarque Woodsworth, les stratégies de traduction et d'écriture sont améliorées du fait de la sensibilisation aux défis et aux mécanismes de transfert interculturel, et de traduction de l'oralité.

Rédigé par Anna Vermeulen de l'Université de Gand et Ana Ibáñez Moreno de l'Université nationale d'enseignement à distance à Madrid, le dernier et quatrième chapitre de la deuxième partie de cet ouvrage porte le titre Audio Description as a Tool to Enhance Intercultural Competence. Ces auteures exposent les résultats d'études de réception entreprises dans le cadre du projet «Audiodescripción como Recurso Didáctico en la Enseñanza de Lenguas Extranjeras» (ARDELE) du Département de traduction, d'interprétation et de communication de la Faculté des arts et de philosophie de l'Université de Gand sur une période 
de trois années scolaires, à savoir de 2011 à 2014. Sur la base des résultats obtenus, Vermeulen et Moreno tirent la conclusion que l'audiodescription constitue un outil pédagogique privilégié pour améliorer la sensibilisation interculturelle, car elle aiguise chez les étudiants le réflexe d'utiliser la langue pour communiquer de manière efficace.

Les quatre chapitres de la troisième et dernière partie sont réunis sous le titre The Assessment of Transcultural Competence in Translation. Dans le premier chapitre, Teaching and Assessing Intercultural Competence: From Common Ground to Divergence, Catherine Way de l'Université de Grenade aborde la problématique liée au développement de la compétence interculturelle qui, selon l'auteure, est souvent limité à la formation initiale du traducteur. Elle prétend que le développement de la compétence interculturelle devrait être aussi intégré aux dernières étapes de la formation du traducteur afin de systématiser la prise de décision en ce qui concerne les problèmes culturels. Way propose également des exemples pour démontrer une façon dont la compétence interculturelle peut être intégrée au système d'évaluation par le biais des rubriques.

Le chapitre deux porte comme titre Intercultural Errors and Translation Assessment: Two Small German-English Case Studies. Geoffrey S. Koby de la Kent State University présente les résultats d'une étude centrée sur les erreurs qui relèvent de la méconnaissance des éléments culturels dans les textes sources. Il s'agit de situations d'examen réelles où 27 traductions d'étudiants de baccalauréat et 28 traductions de candidats d'examen de certification de l'American Translators Association (ATA) de l'année 2006 ont été choisies. La comparaison des deux groupes révèle une similarité de fréquence et de gravité d'erreurs relatives aux éléments culturels. L'auteur considère que ces résultats démontrent la nécessité d'établir des catégories d'erreur plus détaillées en pédagogie de la traduction et d'effectuer plus d'études sur des corpus plus larges tirés des examens de l'ATA.

Dans le troisième chapitre, Cultural Competence in Translation Studies and its Assessment, June Eyckmans de l'Université de Gand examine le rôle accordé à la compétence culturelle dans les différents modèles de compétence de traduction en traductologie: PACTE 2000, Arango-Keith et Koby (2003), Angelelli (2009), ISO 17100 (2015). Elle constate que la plupart des conceptualisations contemporaines de la compétence de traduction adoptent une approche fonctionnelle plutôt que linguistique. En outre, l'auteure s'interroge sur le lien entre les dimensions linguistiques et la compétence culturelle dans le cadre de l'acquisition de la langue seconde et en traductologie. Enfin, Eyckmans porte le regard sur les pratiques d'évaluation actuelles en traduction et passe en revue les propositions pour l'évaluation de la compétence culturelle en tant que sous-compétence de la compétence de traduction.

Dans le quatrième et dernier chapitre intitulé How Does a Study-Abroad Experience Contribute to the Intercultural Competence of Student Translators?, Alexandra Rosiers de l'Université de Gand présente le rapport d'une étude menée à l'aide du Multicultural Personality Questionnaire (MPQSF) pour mesurer le niveau de la compétence interculturelle en amont et en aval du programme d'échange Erasmus. Il s'agit d'une approche sociopsychologique destinée à mesurer l'efficacité multiculturelle qui permet d'évaluer cinq dimensions de personnalité: empathie culturelle, initiative sociale, flexibilité, ouverture d'esprit et stabilité émotionnelle. Sur la base des résultats obtenus, Rosiers conclut qu'un séjour obligatoire à l'étranger intégré à un programme de traduction paraît favorable à la sensibilité culturelle, et plus précisément à la souplesse et l'ouverture d'esprit chez l'étudiant.

Pour les chercheurs qui s'intéressent au développement de la traductologie en tant que jeune discipline connaissant des problèmes méthodologiques et terminologiques, les réflexions théoriques de la première partie seront très motivantes. Les pédagogues qui cherchent souvent à connaître les méthodes adoptées par leurs collègues dans des cours de traduction trouveront bien intéressantes les activités d'apprentissage décrites dans la deuxième partie. Si l'évaluation en traduction reste un sujet peu développé, les résultats des études sur l'évaluation de la compétence culturelle proposés dans la dernière partie de cet ouvrage ouvrent la voie à des pistes de recherche.

Cet ouvrage ne traite pas de la compétence culturelle en général; tout au contraire, les différents chapitres décrivent des situations très spécifiques, offrant ainsi un vaste panorama de perspectives en rapport avec la compétence culturelle en pédagogie de traduction.

Kornebari B. Kumbe

Université de Montréal, Montréal, Canada

\section{RÉFÉRENCE}

Holmes, James S. (1972/1988): The name and nature of Translation Studies. In: James S. HoLmes. Translated!: papers on literary translation and translation studies. Amsterdam: Rodopi, 67-80. 